\title{
Symmetric chain decomposition of necklace posets
}

\author{
Vivek Dhand \\ vivek. dhand@gmail.com
}

\author{
Submitted: Jun 24, 2011; Accepted: Jan 12, 2012; Published: Jan 21, 2012 \\ Mathematics Subject Classification: 05E18, 06A07
}

\begin{abstract}
A finite ranked poset is called a symmetric chain order if it can be written as a disjoint union of rank-symmetric, saturated chains. If $\mathcal{P}$ is any symmetric chain order, we prove that $\mathcal{P}^{n} / \mathbb{Z}_{n}$ is also a symmetric chain order, where $\mathbb{Z}_{n}$ acts on $\mathcal{P}^{n}$ by cyclic permutation of the factors.
\end{abstract}

\section{Introduction}

Let $(\mathcal{P},<)$ be a finite poset. A chain in $\mathcal{P}$ is a sequence of the form $x_{1}<x_{2}<\cdots<x_{n}$ where each $x_{i} \in \mathcal{P}$. For $x, y \in \mathcal{P}$, we say $y$ covers $x$ (denoted $x \lessdot y$ ) if $x<y$ and there does not exist $z \in \mathcal{P}$ such that $x<z$ and $z<y$. A saturated chain in $\mathcal{P}$ is a chain where each element is covered by the next. We say $\mathcal{P}$ is ranked if there exists a function rk $: \mathcal{P} \rightarrow \mathbb{Z}_{\geq 0}$ such that $x \lessdot y$ implies $\operatorname{rk}(y)=\operatorname{rk}(x)+1$. The rank of $\mathcal{P}$ is defined as $\operatorname{rk}(\mathcal{P})=\max \{\operatorname{rk}(x) \mid x \in \mathcal{P}\}+\min \{\operatorname{rk}(x) \mid x \in \mathcal{P}\}$. A saturated chain $\left\{x_{1} \lessdot x_{2} \lessdot \cdots \lessdot x_{n}\right\}$ in a ranked poset $\mathcal{P}$ is said to be rank-symmetric if $\operatorname{rk}\left(x_{1}\right)+\operatorname{rk}\left(x_{n}\right)=\operatorname{rk}(\mathcal{P})$.

We say that $\mathcal{P}$ has a symmetric chain decomposition if it can be written as a disjoint union of saturated, rank-symmetric chains. A symmetric chain order is a finite ranked poset for which there exists a symmetric chain decomposition.

A finite product of symmetric chain orders is a symmetric chain order. This result can be proved by induction [1] or by explicit constructions (e.g. [3]). Naturally, this raises the question of whether the quotient of a symmetric chain order under a given group action has a symmetric chain decomposition. For example, if $X$ is a set then $\mathbb{Z}_{n}$ acts on the set $\operatorname{Map}\left(\mathbb{Z}_{n}, X\right) \simeq X^{n}$. The elements of $X^{n} / \mathbb{Z}_{n}$ are called $n$-bead necklaces with labels in $X$. A symmetric chain decomposition of the poset of binary necklaces was first constructed by K. Jordan [6], building on the work of Griggs-Killian-Savage [4]. There have been recent independent proofs and generalizations of these results $[2,5]$. The main result of this paper is the following:

Theorem 1.1. If $\mathcal{P}$ is a symmetric chain order, then $\mathcal{P}^{n} / \mathbb{Z}_{n}$ is a symmetric chain order. 
We give a brief outline of the proof. First, we show that the poset of $n$-bead binary necklaces is isomorphic to the poset of partition necklaces, i.e. $n$-bead necklaces labeled by positive integers which sum to $n$. It turns out to be convenient to exclude the maximal and minimal binary necklaces, which correspond to those partitions of $n$ having $n$ parts and 0 parts, respectively. Let $\mathcal{Q}(n)$ denote the poset of partition necklaces with these two elements removed. We decompose $\mathcal{Q}(n)$ into rank-symmetric sub-posets $\mathcal{Q}_{\alpha}$, running over partition necklaces $\alpha$ where 1 does not appear. This decomposition corresponds to the "block-code" decomposition of binary necklaces defined in [4].

We can also extend this idea to non-binary necklaces. In fact, the poset of $n$-bead $(m+1)$-ary necklaces embeds into the poset of $n m$-bead binary necklaces, and the image corresponds to the union of those $Q_{\alpha} \subset \mathcal{Q}(m n)$ such that every part of $\alpha$ is divisible by $m$.

Next, we prove a "factorization property" for $Q_{\alpha} \subset \mathcal{Q}(n)$. If $P$ and $Q$ are finite ranked posets, we say that $P$ covers $Q$ (or $Q$ is covered by $P$ ) if there is a morphism of ranked posets from $P$ to $Q$ which is a bijection on the underlying sets. We denote this relation as $P \hookrightarrow Q$. Note that any ranked poset covered by a symmetric chain order is also a symmetric chain order. If $\alpha$ is aperiodic, then $Q_{\alpha}$ is covered by a product of symmetric chains. If $\alpha$ is periodic of period $d$, then $Q_{\alpha}$ is covered by the poset of $(n / d)$-bead necklaces labeled by $\mathcal{Q}_{\beta}$, for some aperiodic $d$-bead necklace $\beta$.

Finally, if $\mathcal{P}$ is a symmetric chain order, then $\mathcal{P}^{n} / \mathbb{Z}_{n}$ has a decomposition into posets which are either products of chains, or posets of $d$-bead necklaces with labels in a product of chains (where $d<n$ ), or posets of $n$-bead $(m+1)$-ary necklaces for some $m \geq 1$. In each case, we apply induction to finish the proof.

\section{Generalities on necklaces}

We begin by recalling some basic facts about $\mathbb{Z}_{n}$-actions on sets. We will use additive notation for the group operation of $\mathbb{Z}_{n}$. The subgroups of $\mathbb{Z}_{n}$ are of the form $\langle d\rangle$ where $d$ is a positive divisor of $n$, and $\mathbb{Z}_{n} /\langle d\rangle \simeq \mathbb{Z}_{d}$. If $X$ is a set with $\mathbb{Z}_{n}$-action, let $X^{\langle d\rangle}$ denote the set of $\langle d\rangle$-fixed points in $X$. Equivalently:

$$
X^{\langle d\rangle}=\left\{x \in X \mid\langle d\rangle \subset S t a b_{\mathbb{Z}_{n}}(x)\right\} .
$$

Note that $X^{\langle c\rangle} \subset X^{\langle d\rangle}$ if $c$ is a divisor of $d$. Next, we define:

$$
X^{\{d\}}=\left\{x \in X \mid\langle d\rangle=\operatorname{Stab}_{\mathbb{Z}_{n}}(x)\right\} .
$$

Of course, we have:

$$
X=\bigsqcup_{d \mid n} X^{\{d\}}
$$

and the $\mathbb{Z}_{n}$ action on $X^{\{d\}}$ factors through $\mathbb{Z}_{d}$. In other words, we have a bijection:

$$
X / \mathbb{Z}_{n} \simeq \bigsqcup_{d \mid n} X^{\{d\}} / \mathbb{Z}_{d}
$$


Now consider the special case where $X=\operatorname{Map}\left(\mathbb{Z}_{n}, Y\right)$ for some arbitrary set $Y$, where $\mathbb{Z}_{n}$ acts on the first factor. In other words,

$$
(a f)(b)=f(a+b)
$$

for any $a, b \in \mathbb{Z}_{n}$ and $f: \mathbb{Z}_{n} \rightarrow Y$. Now the previous paragraph implies that:

$$
\operatorname{Map}\left(\mathbb{Z}_{n}, Y\right)=\bigsqcup_{d \mid n} \operatorname{Map}\left(\mathbb{Z}_{n}, Y\right)^{\{d\}}
$$

and

$$
\operatorname{Map}\left(\mathbb{Z}_{n}, Y\right) / \mathbb{Z}_{n}=\bigsqcup_{d \mid n} \operatorname{Map}\left(\mathbb{Z}_{n}, Y\right)^{\{d\}} / \mathbb{Z}_{d}
$$

The elements of $\operatorname{Map}\left(\mathbb{Z}_{n}, Y\right) / \mathbb{Z}_{n}$ are called $n$-bead necklaces with labels in $Y$. An element of $\operatorname{Map}\left(\mathbb{Z}_{n}, Y\right)^{\{d\}} / \mathbb{Z}_{d}$ is said to be periodic of period d. An element of $\operatorname{Map}\left(\mathbb{Z}_{n}, Y\right)^{\{n\}} / \mathbb{Z}_{n}$ is said to be aperiodic. Given a map $g: \mathbb{Z}_{n} \rightarrow Y$, let $[g]$ denote the corresponding necklace in $\operatorname{Map}\left(\mathbb{Z}_{n}, Y\right) / \mathbb{Z}_{n}$. A $n$-bead necklace with labels in $Y$ can be visualized as a sequence of $n$ elements of $Y$ placed evenly around a circle, where we discount the effect of rotation by any multiple of $\frac{2 \pi}{n}$ radians. Given $\left(y_{1}, \ldots, y_{n}\right) \in Y^{n}$, let $\left[y_{1}, \ldots, y_{n}\right]$ denote the corresponding $n$-bead necklace.

Our first observation is that an $n$-bead necklace of period $d$ is uniquely determined by any sequence of $d$ consecutive elements around the circle. Moreover, as we rotate the circle, these $d$ elements will behave exactly like an aperiodic $d$-bead necklace.

Proposition 2.1. There is a natural bijection between $n$-bead necklaces of period $d$ and aperiodic $d$-bead necklaces.

Proof. Recall the following general fact: if $G$ is a group, $H$ is a normal subgroup of $G$, and $Y$ is an arbitrary set, then there is an isomorphism of $G$-sets:

$$
\begin{gathered}
\operatorname{Map}(G, Y)^{H} \simeq \operatorname{Map}(G / H, Y) \\
f \mapsto(g H \mapsto f(g)) .
\end{gathered}
$$

Moreover, the action of $G$ on each side factors through $G / H$. In particular, there is an isomorphism of $\mathbb{Z}_{n}$-sets:

$$
\operatorname{Map}\left(\mathbb{Z}_{n}, Y\right)^{\langle d\rangle} \simeq \operatorname{Map}\left(\mathbb{Z}_{d}, Y\right)
$$

where the $\mathbb{Z}_{n}$-action factors through $\mathbb{Z}_{d}$. Looking at elements of period $d$, we get:

$$
\operatorname{Map}\left(\mathbb{Z}_{n}, Y\right)^{\{d\}} \simeq \operatorname{Map}\left(\mathbb{Z}_{d}, Y\right)^{\{d\}}
$$

and so:

$$
\operatorname{Map}\left(\mathbb{Z}_{n}, Y\right)^{\{d\}} / \mathbb{Z}_{d} \simeq \operatorname{Map}\left(\mathbb{Z}_{d}, Y\right)^{\{d\}} / \mathbb{Z}_{d}
$$


Now suppose that $Y$ is a disjoint union of non-empty subsets:

$$
Y=\bigsqcup_{i \in I} Y_{i}
$$

where $I$ is a finite set. Equivalently, we have a surjective map $\pi: Y \rightarrow I$, where $Y_{i}=\pi^{-1}(i)$ for each $i \in I$. It follows that there is a surjective map:

$$
\begin{gathered}
\pi_{*}: \operatorname{Map}\left(\mathbb{Z}_{n}, Y\right) \rightarrow \operatorname{Map}\left(\mathbb{Z}_{n}, I\right) \\
\pi_{*}(f)=\pi \circ f .
\end{gathered}
$$

Given a map $g: \mathbb{Z}_{n} \rightarrow I$, we define:

$$
\operatorname{Map}\left(\mathbb{Z}_{n}, Y\right)=\pi_{*}^{-1}(g)=\left\{f: \mathbb{Z}_{n} \rightarrow Y \mid \pi \circ f=g\right\} .
$$

In other words, $f \in \operatorname{Map}_{g}\left(\mathbb{Z}_{n}, Y\right)$ if and only if $f(a) \in Y_{g(c)}$ for all $a \in \mathbb{Z}_{n}$. Since $\pi_{*}$ is surjective, we have a decomposition:

$$
\operatorname{Map}\left(\mathbb{Z}_{n}, Y\right)=\bigsqcup_{g \in \operatorname{Map}\left(\mathbb{Z}_{n}, I\right)} \operatorname{Map}_{g}\left(\mathbb{Z}_{n}, Y\right)
$$

Note that $\operatorname{Map}_{g}\left(\mathbb{Z}_{n}, Y\right)$ is not necessarily stable under the action of $\mathbb{Z}_{n}$. If $a, b \in \mathbb{Z}_{n}$ and $f \in \operatorname{Map}_{g}\left(\mathbb{Z}_{n}, Y\right)$, then:

$$
a(f)(b)=f(a+b) \in Y_{g(a+b)}
$$

so we have a bijection:

$$
\operatorname{Map}_{g}\left(\mathbb{Z}_{n}, Y\right) \simeq \operatorname{Map}_{a g}\left(\mathbb{Z}_{n}, Y\right)
$$

induced by the action of $a \in \mathbb{Z}_{n}$. We define:

$$
\operatorname{Map}_{[g]}\left(\mathbb{Z}_{n}, Y\right)=\bigcup_{a \in \mathbb{Z}_{n}} \operatorname{Map}_{a g}\left(\mathbb{Z}_{n}, Y\right)
$$

Note that $\mathbb{Z}_{n}$ acts on $\operatorname{Map}_{[g]}\left(\mathbb{Z}_{n}, Y\right)$.

Remark 2.2. We recall a basic observation which will make it easier to define maps on sets of necklaces. Suppose $S$ and $T$ are sets equipped with equivalence relations $\sim$ and $\approx$, respectively. Let $U$ be a subset of $S$ which has a non-trivial intersection with each equivalence class in $S$. Then $U$ inherits the equivalence relation $\sim$ and the natural map from $U / \sim$ to $S / \sim$ is a bijection. Given a map $f: U \rightarrow T$ such that $u_{1} \sim u_{2} \Longrightarrow f\left(u_{1}\right) \approx$ $f\left(u_{2}\right)$ for all $u_{1}, u_{2} \in U$, we obtain a $\operatorname{map}(S / \sim) \simeq(U / \sim) \rightarrow(T / \approx)$.

Remark 2.3. If $\alpha$ is a periodic $n$-bead necklace of period $d$ with labels in $I$, then:

$$
\alpha=[\underbrace{\beta, \ldots, \beta}_{\frac{n}{d} \text { times }}]
$$

where $\beta=\left(\beta_{1}, \ldots, \beta_{d}\right)$ is a $d$-tuple of elements in $I$ such that $[\beta]$ is aperiodic. 
Lemma 2.4. Let $\pi: Y \rightarrow I$ be a surjective map where $I$ is finite.

(1) There is a natural decomposition:

$$
\operatorname{Map}\left(\mathbb{Z}_{n}, Y\right) / \mathbb{Z}_{n}=\bigsqcup_{d \mid n}\left(\bigsqcup_{\alpha \in M a p\left(\mathbb{Z}_{n}, I\right)^{\{d\}} / \mathbb{Z}_{d}} \operatorname{Map}_{\alpha}\left(\mathbb{Z}_{n}, Y\right) / \mathbb{Z}_{n}\right)
$$

(2) If $\alpha=[\beta, \ldots, \beta] \in \operatorname{Map}\left(\mathbb{Z}_{n}, I\right)^{\{d\}} / \mathbb{Z}_{d}$, where $\beta=\left(\beta_{1}, \ldots, \beta_{d}\right)$, then there is a bijection:

$$
\operatorname{Map}_{\alpha}\left(\mathbb{Z}_{n}, Y\right) / \mathbb{Z}_{n} \simeq\left(Y_{\beta_{1}} \times \cdots \times Y_{\beta_{d}}\right)^{\frac{n}{d}} / \mathbb{Z}_{\frac{n}{d}}
$$

Proof. (1) Since

$$
\operatorname{Map}\left(\mathbb{Z}_{n}, Y\right)=\bigsqcup_{g \in \operatorname{Map}\left(\mathbb{Z}_{n}, I\right)} \operatorname{Map}\left(\mathbb{Z}_{n}, Y\right)
$$

and

$$
\operatorname{Map}\left(\mathbb{Z}_{n}, I\right)=\bigsqcup_{d \mid n} \operatorname{Map}\left(\mathbb{Z}_{n}, I\right)^{\{d\}}
$$

we see that:

$$
\operatorname{Map}\left(\mathbb{Z}_{n}, Y\right)=\bigsqcup_{d \mid n}\left(\bigsqcup_{g \in M a p\left(\mathbb{Z}_{n}, I\right)^{\{d\}}} \operatorname{Map}_{g}\left(\mathbb{Z}_{n}, Y\right)\right)
$$

As noted above, in order to make this an equality of $\mathbb{Z}_{n}$-sets we need to take the coarser decomposition:

$$
\operatorname{Map}\left(\mathbb{Z}_{n}, Y\right)=\bigsqcup_{d \mid n}\left(\bigsqcup_{[g] \in M a p\left(\mathbb{Z}_{n}, I\right)\{d\} / \mathbb{Z}_{d}} \operatorname{Map}_{[g]}\left(\mathbb{Z}_{n}, Y\right)\right)
$$

Now we simply take the quotient by $\mathbb{Z}_{n}$ on both sides:

$$
\operatorname{Map}\left(\mathbb{Z}_{n}, Y\right) / \mathbb{Z}_{n}=\bigsqcup_{d \mid n}\left(\bigsqcup_{[g] \in \operatorname{Map}\left(\mathbb{Z}_{n}, I\right)^{\{d\}} / \mathbb{Z}_{d}} \operatorname{Map}_{[g]}\left(\mathbb{Z}_{n}, Y\right) / \mathbb{Z}_{n}\right)
$$

Note that we are simply organizing the $n$-bead $Y$-labeled necklaces by looking at the periods of the underlying $n$-bead $I$-labeled necklaces.

(2) Let $g \in \operatorname{Map}\left(\mathbb{Z}_{n}, I\right)^{\{d\}}$ and let $a \in \mathbb{Z}_{n}$. By definition, $a g=(a+x) g$ if and only if $x \in\langle d\rangle$. So:

$$
\operatorname{Map}_{a g}\left(\mathbb{Z}_{n}, Y\right)=\operatorname{Map}_{(a+x) g}\left(\mathbb{Z}_{n}, Y\right)
$$

if $x \in\langle d\rangle$. On the other hand, if

$$
h \in \operatorname{Map}_{a g}\left(\mathbb{Z}_{n}, Y\right) \cap \operatorname{Map}_{(a+x) g}\left(\mathbb{Z}_{n}, Y\right)
$$


for some $x \in \mathbb{Z}_{n}$, then $\pi \circ h=a g=(a+x) g$, which implies that $x \in\langle d\rangle$. The upshot is that we can actually write $\operatorname{Map}_{[g]}\left(\mathbb{Z}_{n}, Y\right)$ as a disjoint union over $\mathbb{Z}_{d}$ :

$$
\operatorname{Map}_{[g]}\left(\mathbb{Z}_{n}, Y\right)=\bigsqcup_{a \in \mathbb{Z}_{d}} \operatorname{Map}_{a g}\left(\mathbb{Z}_{n}, Y\right)
$$

Now consider the sequence of values $g(a)$ for $a \in \mathbb{Z}_{n}$. This sequence is of the form $(\beta, \ldots, \beta)$, where $\beta=\left(\beta_{1}, \ldots, \beta_{d}\right)$. Therefore:

$$
\operatorname{Map}_{g}\left(\mathbb{Z}_{n}, Y\right) \simeq\left(Y_{\beta_{1}} \times \cdots \times Y_{\beta_{d}}\right)^{\frac{n}{d}}
$$

and so:

$$
\operatorname{Map}_{[g]}\left(\mathbb{Z}_{n}, Y\right) \simeq \bigsqcup_{j=0}^{d-1}\left(Y_{\beta_{j+1}} \times \cdots \times Y_{\beta_{d}} \times Y_{\beta_{1}} \times \cdots \times Y_{\beta_{j}}\right)^{\frac{n}{d}}
$$

Let us apply Remark 2.2 to the following sets:

$$
S=\bigsqcup_{j=0}^{d-1}\left(Y_{\beta_{j+1}} \times \cdots \times Y_{\beta_{d}} \times Y_{\beta_{1}} \times \cdots \times Y_{\beta_{j}}\right)^{\frac{n}{d}} \quad \text { and } \quad T=\left(Y_{\beta_{1}} \times \cdots \times Y_{\beta_{d}}\right)^{\frac{n}{d}}
$$

The equivalence relations on $S$ and $T$ are defined by group actions: $\mathbb{Z}_{n}$ acts on $S \simeq$ $\operatorname{Map}_{[g]}\left(\mathbb{Z}_{n}, Y\right)$ and $\mathbb{Z}_{\frac{n}{d}}$ acts on $T$ by cyclic permutation of the factors. Let $U$ be the subset of $S$ corresponding to the $j=0$ component:

$$
U=\left(Y_{\beta_{1}} \times \cdots \times Y_{\beta_{d}}\right)^{\frac{n}{d}} .
$$

Each element of $S$ is equivalent to an element of $U$, and the restricted equivalence relation on $U$ is given by the action of the subgroup $\langle d\rangle$ which is exactly the same as the action of $\mathbb{Z}_{\frac{n}{d}}$ by cyclic permutation of the factors. Therefore:

$$
S / \mathbb{Z}_{n} \simeq U /\langle d\rangle \simeq T / \mathbb{Z}_{\frac{n}{d}}
$$

Remark 2.5. We can visualize the above result as follows: we choose a place to "cut" an $n$-bead $Y$-labeled necklace in order to get an $n$-tuple of elements of $Y$. We can always rotate the original necklace so that the underlying $I$-labeled necklace has a given position with respect to the cut. Moreover, if the underlying $I$-labeled necklace has period $d$, then we can break the $n$-tuple into segments of size $d$ so that the corresponding $I$-labeled $d$-bead necklaces are aperiodic. As we rotate the original necklace by multiples of $\frac{2 \pi}{d}$ radians, we will permute these segments among each other. 


\section{Partition necklaces}

Let $n$ be a positive integer. Consider the set of ordered partitions of $n$ into $r$ positive parts:

$$
\mathcal{P}(n, r)=\left\{\left(a_{1}, \ldots, a_{r}\right) \in \mathbb{Z}_{>0}^{r} \mid \sum_{i=1}^{r} a_{i}=n\right\}
$$

Define:

$$
\mathcal{P}(n)=\bigsqcup_{r=1}^{n-1} \mathcal{P}(n, r)
$$

In other words, $\mathcal{P}(n)$ is the set of non-empty ordered partitions of $n$ into positive parts, where at least one part is greater than 1 . Note that refinement of partitions defines a partial order on $\mathcal{P}(n)$, and the rank of a partition is given by the number of parts.

Let $\mathcal{Q}(n)$ denote the set of necklaces associated to $\mathcal{P}(n)$ :

$$
\mathcal{Q}(n)=\bigsqcup_{i=1}^{n-1} \mathcal{P}(n, r) / \mathbb{Z}_{r}
$$

In other words:

$$
\mathcal{Q}(n)=\left\{\left[a_{1}, \ldots, a_{r}\right] \in \mathbb{Z}_{>0}^{r} / \mathbb{Z}_{r} \mid 1 \leq r \leq n-1, \sum_{i=1}^{r} a_{i}=n\right\}
$$

where $\left[a_{1}, \ldots, a_{r}\right]$ denotes the $\mathbb{Z}_{r}$-orbit of $\left(a_{1}, \ldots, a_{r}\right)$.

The elements of $\mathcal{Q}(n)$ are called partition necklaces. Note that $\mathcal{Q}(n)$ inherits the structure of a ranked poset from $\mathcal{P}(n)$.

Let $\mathcal{N}(n, 1)$ denote the set of $n$-bead binary necklaces with the necklaces $[0, \ldots, 0]$ and $[1, \ldots, 1]$ removed.

Proposition 3.1. For any $n \geq 1$, there is an isomorphism of ranked posets:

$$
\psi_{n}: \mathcal{N}(n, 1) \simeq \mathcal{Q}(n) .
$$

Proof. Given a non-empty $n$-bead binary necklace $\beta$ of rank $r$, let $\psi_{n}(\beta)$ be the necklace whose entries are given by the number of steps between consecutive non-zero entries of $\beta$. More precisely, $\psi_{n}$ is given by:

$$
\left[1,0^{c_{1}}, 1,0^{c_{2}}, \ldots, 1,0^{c_{r}}\right] \mapsto\left[c_{1}+1, \ldots, c_{r}+1\right]
$$

Note that the right hand side is the necklace of a partition of $n$ into $r$ positive parts. The inverse of $\psi_{n}$ is given by:

$$
\left[a_{1}, \ldots, a_{r}\right] \mapsto\left[1,0^{a_{1}-1}, 1,0^{a_{2}-1}, \ldots, 1,0^{a_{r}-1}\right]
$$

Moreover, changing a "zero" to a "one" in a binary necklace corresponds to a refinement of the corresponding partition necklace, so the above bijection is compatible with the partial orders and rank functions on each poset. 
An ordered partition $\left(a_{1}, \ldots, a_{r}\right)$ and the corresponding partition necklace $\left[a_{1}, \ldots, a_{r}\right]$ are said to be fundamental if each $a_{i} \geq 2$. Let $\mathcal{F}(n)$ denote the set of fundamental partition necklaces in $Q(n)$.

Now we apply Remark 2.2 to the case where $S=\mathcal{P}(n)$ and $T$ is the subset of $\mathcal{P}(n)$ consisting of fundamental partitions. Equip each set with the necklace equivalence relation, so $(S / \sim)=\mathcal{Q}(n)$ and $(T / \approx)=\mathcal{F}(n)$. Define the subset:

$$
U=\left\{\left(1^{n_{1}}, m_{1}, 1^{n_{2}}, m_{2}, \ldots, 1^{n_{k}}, m_{k}\right) \in \mathcal{P}(n) \mid n_{i} \geq 0, m_{i} \geq 2 \text { for all } 1 \leq i \leq k\right\}
$$

Since we have excluded $(1, \ldots, 1)$ from $\mathcal{P}(n)$, we see that any element of $\mathcal{P}(n)$ is equivalent to some element in $U$. Now define:

$$
\begin{aligned}
f: U & \rightarrow T \\
\left(1^{n_{1}}, m_{1}, 1^{n_{2}}, m_{2}, \ldots, 1^{n_{k}}, m_{k}\right) & \mapsto\left(m_{1}+n_{1}, \ldots, m_{k}+n_{k}\right) .
\end{aligned}
$$

Since $f$ is compatible with the respective equivalence relations, we obtain a map:

$$
\begin{aligned}
\pi_{n}: \mathcal{Q}(n) \rightarrow \mathcal{F}(n) \\
{\left[1^{n_{1}}, m_{1}, 1^{n_{2}}, m_{2}, \ldots, 1^{n_{k}}, m_{k}\right] \mapsto\left[m_{1}+n_{1}, m_{2}+n_{2}, \ldots, m_{k}+n_{k}\right] . }
\end{aligned}
$$

Note that $\pi_{n}$ restricts to the identity on $\mathcal{F}(n)$. In particular, $\pi_{n}$ is surjective. Therefore, we get a decomposition of $\mathcal{Q}(n)$ :

$$
\mathcal{Q}(n)=\bigsqcup_{\alpha \in \mathcal{F}(n)} \mathcal{Q}_{\alpha}
$$

where $Q_{\alpha}=\pi_{n}^{-1}(\alpha)$. This decomposition is the same as the decomposition for binary necklaces defined in [4]. Indeed, the map $\pi_{n} \circ \psi_{n}$ is essentially the necklace version of the "block-code" construction.

If $m \geq 1$, a fundamental partition necklace $\left[a_{1}, \ldots, a_{r}\right] \in \mathcal{F}(n)$ is said to be divisible by $m$ if each $a_{i}$ is divisible by $m$. Define the following sub-poset of $Q(n)$ :

$$
\mathcal{Q}(n, m)=\left\{\alpha \in \mathcal{Q}(n) \mid \pi_{n}(\alpha) \text { is divisible by } m\right\}=\bigsqcup_{\substack{\alpha \in \mathcal{F}(n) \\ m \mid \alpha}} \mathcal{Q}_{\alpha} \text {. }
$$

Let $\mathcal{N}(n, m)$ denote the set of $n$-bead $(m+1)$-ary necklaces with the necklaces $[0, \ldots, 0]$ and $[m, \ldots, m]$ removed. We have the following generalization of Proposition 3.1.

Lemma 3.2. For any $n, m \geq 1$, there is an isomorphism of ranked posets:

$$
\psi_{n, m}: \mathcal{N}(n, m) \simeq \mathcal{Q}(m n, m) .
$$


Proof. Given an $n$-bead $(m+1)$-ary necklace, we construct an $m n$-bead binary necklace via the substitution: $j \mapsto 1^{j} 0^{m-j}$, and then we apply the map $\psi_{m n}$ from Proposition 3.1. This composition is clearly a morphism of ranked posets. Here is an explicit formula for $\psi_{n, m}$ :

$$
\left[b_{1}, 0^{c_{1}}, b_{2}, 0^{c_{2}}, \ldots, b_{r}, 0^{c_{r}}\right] \mapsto\left[1^{b_{1}-1}, m\left(c_{1}+1\right)-b_{1}+1, \ldots, 1^{b_{r}-1}, m\left(c_{r}+1\right)-b_{r}+1\right]
$$

where each $b_{i} \geq 1$ and $c_{i} \geq 0$. The sum of the terms in the partition necklace is:

$$
\sum_{i=1}^{r}\left(b_{i}-1+m\left(c_{i}+1\right)-b_{i}+1\right)=m\left(r+\sum_{i=1}^{r} c_{i}\right)=m n
$$

as desired. Let us check that $\pi_{m n} \circ \psi_{n, m}(\alpha)$ is divisible by $m$ for all $\alpha \in \mathcal{N}(n, m)$. Consider the element:

$$
\alpha=\left[b_{1}, 0^{c_{1}}, b_{2}, 0^{c_{2}}, \ldots, b_{r}, 0^{c_{r}}\right] .
$$

If $c_{i}>0$ or $b_{i}<m$, then the terms $1^{b_{i}-1}$ and $m\left(c_{i}+1\right)-b_{i}+1$ in $\psi_{m, n}(\alpha)$ merge together under $\pi_{m n}$ to give $m\left(c_{i}+1\right)$. On the other hand, whenever $b_{i}=m$ and $c_{i}=0$, we will get a $1^{m}$ term in $\psi_{m, n}(\alpha)$. Applying $\pi_{m n}$ will result in adding $m$ to the next occurrence of $m\left(c_{j}+1\right)$, where $c_{j}>1$. In other words:

$$
\pi_{m n}\left(\psi_{n, m}(\alpha)\right)=\left[m e_{1}, \ldots, m e_{s}\right]
$$

where $\pi_{n}\left(c_{1}+1, \ldots, c_{r}+1\right)=\left[e_{1}, \ldots, e_{s}\right]$, and this result is indeed divisible by $m$.

By reversing the above process, we get a formula for the inverse of $\psi_{n, m}$. An arbitrary element of $\mathcal{Q}(m n, m)$ is of the form:

$$
\left[1^{n_{1}}, m_{1}, 1^{n_{2}}, m_{2}, \ldots, 1^{n_{k}}, m_{k}\right]
$$

where each $m_{i} \geq 2$, each $m_{i}+n_{i}$ is divisible by $m$, and $\sum_{i=1}^{k}\left(m_{i}+n_{i}\right)=m n$. The corresponding $m n$-bead binary necklace is:

$$
\left[1^{n_{1}+1}, 0^{m_{1}-1}, \ldots, 1^{n_{k}+1}, 0^{m_{k}-1}\right]
$$

Now we need to apply the substitution $1^{j} 0^{m-j} \mapsto j$. Since $m_{i}+n_{i}$ is divisible by $m$, we can apply this to each block $\left(1^{n_{i}+1}, 0^{m_{i}-1}\right)$ separately. Furthermore, we should break each block into segments of size $m$ and apply the substitution to each segment. Therefore, $\left(1^{n_{i}+1}, 0^{m_{i}-1}\right)$ looks like:

$$
(\underbrace{1^{m}, 1^{m}, \ldots, 1^{m}}_{q_{i} \text { times }}, 1^{r_{i}}, 0^{m-r_{i}}, 0^{m_{i}-1-\left(m-r_{i}\right)}) .
$$

where $q_{i}$ is the quotient of the division of $n_{i}+1$ by $m$ and $r_{i}$ is the remainder. Note that $m_{i}-1-\left(m-r_{i}\right)=m_{i}-1-m+\left(n_{i}+1-m q_{i}\right)=m_{i}+n_{i}-m q_{i}-m$, which is divisible by $m$. Therefore, the inverse of $\psi_{n, m}$ is given by the following formula:

$$
\left[1^{n_{1}}, m_{1}, 1^{n_{2}}, m_{2}, \ldots, 1^{n_{k}}, m_{k}\right] \mapsto\left[m^{q_{1}}, r_{1}, 0^{t_{1}} \ldots, m^{q_{k}}, r_{k}, 0^{t_{k}}\right]
$$


where:

$$
n_{i}+1=m q_{i}+r_{i} \text { such that } 0 \leq r_{i}<m
$$

and

$$
t_{i}=\frac{m_{i}+n_{i}}{m}-q_{i}-1
$$

Note that the number of beads in the above necklace is:

$$
\sum_{i=1}^{k}\left(q_{i}+1+\frac{m_{i}+n_{i}}{m}-q_{i}-1\right)=\frac{1}{m} \sum_{i=1}^{k}\left(m_{i}+n_{i}\right)=\frac{m n}{m}=n
$$

as desired.

Lemma 3.3. Let $\alpha=\left[a_{1}, \ldots, a_{r}\right] \in \mathcal{F}(n)$. If $\alpha$ is aperiodic, then:

$$
\mathcal{Q}_{\left[a_{1}\right]} \times \cdots \times \mathcal{Q}_{\left[a_{r}\right]} \hookrightarrow \mathcal{Q}_{\alpha} .
$$

If $\alpha$ is periodic of period $d$ and $\alpha=[\underbrace{\beta, \ldots, \beta}_{\frac{r}{d} \text { times }}]$, then:

$$
\mathcal{Q}_{[\beta]}^{\frac{r}{d}} / \mathbb{Z}_{\frac{r}{d}} \hookrightarrow Q_{\alpha}
$$

Proof. If $m \geq 2$, note that $\mathcal{Q}_{[m]}$ is a chain with $m-1$ vertices. We will apply Lemma 2.4 to the following set:

$$
\mathcal{Q}=\bigsqcup_{m=2}^{n} \mathcal{Q}_{[m]}
$$

Note that our indexing set is $I=\{2, \ldots, n\}$. Let $\alpha=\left[a_{1}, \ldots, a_{r}\right] \in \mathcal{F}(n)$. Since $a_{1}+$ $\cdots+a_{r}=n$, we know that each $a_{i} \leq n$, which implies that $\alpha$ is labeled by elements of $I$. If $\alpha$ is aperiodic, it follows from part (2) of Lemma 2.4 that we have a rank-preserving bijection:

$$
\operatorname{Map}_{\alpha}\left(\mathbb{Z}_{r}, \mathcal{Q}\right) / \mathbb{Z}_{r} \simeq \mathcal{Q}_{\left[a_{1}\right]} \times \cdots \times \mathcal{Q}_{\left[a_{r}\right]} .
$$

On the other hand, if $\alpha=[\beta, \ldots, \beta] \in \operatorname{Map}\left(\mathbb{Z}_{r}, I\right)^{\{d\}} / \mathbb{Z}_{d}$, where $\beta=\left(\beta_{1}, \ldots, \beta_{d}\right)$, then we have rank-preserving bijections:

$$
\operatorname{Map}_{\alpha}\left(\mathbb{Z}_{r}, Q\right) / \mathbb{Z}_{r} \simeq\left(\mathcal{Q}_{\left[\beta_{1}\right]} \times \cdots \times \mathcal{Q}_{\left[\beta_{d}\right]}\right)^{\frac{r}{d}} / \mathbb{Z}_{\frac{r}{d}} \simeq \mathcal{Q}_{[\beta]}^{\frac{r}{d}} / \mathbb{Z}_{\frac{r}{d}}
$$

where the second bijection exists due to the fact that $[\beta]$ is aperiodic. It remains to check that the poset relations are preserved. Indeed, any covering relation among two necklaces labeled by $Q_{\left[\beta_{1}\right]} \times \cdots \times Q_{\left[\beta_{d}\right]}$ will correspond to a covering relation within a chain $\mathcal{Q}_{\left[\beta_{i}\right]}$ for some $i$, which will also be a covering relation among the corresponding Q-labeled necklaces.

Remark 3.4. The above Lemma provides an explanation of why it is easier to find a symmetric chain decomposition of $n$-bead binary necklaces if $n$ in prime [4]. Indeed, in this case all non-trivial necklaces are aperiodic, so each $Q_{\alpha}$ is covered by a product of symmetric chains and we can apply the Greene-Kleitman rule. 


\section{Proof of the theorem}

Theorem 4.1. If $\mathcal{P}$ is a symmetric chain order, then $\mathcal{P}^{n} / \mathbb{Z}_{n}$ is a symmetric chain order.

Proof. The statement is trivial for $n=1$. Assume that the theorem is true for any $n^{\prime}<n$. Let $C_{1}, \ldots, C_{r}$ denote the chains in a symmetric chain decomposition of $\mathcal{P}$. We may assume that:

$$
\mathcal{P}=\bigsqcup_{i=1}^{r} C_{i}
$$

If we let $I=\{1,2, \ldots, r\}$ and apply part (1) of Lemma 2.4 to $\mathcal{P}$, we obtain:

$$
\operatorname{Map}\left(\mathbb{Z}_{n}, \mathcal{P}\right) / \mathbb{Z}_{n}=\bigsqcup_{d \mid n}\left(\bigsqcup_{\alpha \in M a p\left(\mathbb{Z}_{n}, I\right)^{\{d\}} / \mathbb{Z}_{d}} \operatorname{Map}_{\alpha}\left(\mathbb{Z}_{n}, \mathcal{P}\right) / \mathbb{Z}_{n}\right)
$$

Now we apply part (2) of Lemma 2.4. If $\alpha=\left[a_{1}, \ldots, a_{n}\right]$ is an aperiodic $n$-bead necklace with labels in $I$, then:

$$
C_{a_{1}} \times \cdots \times C_{a_{n}} \hookrightarrow \operatorname{Map}_{\alpha}\left(\mathbb{Z}_{n}, \mathcal{P}\right)
$$

Since $C_{a_{1}} \times \cdots \times C_{a_{n}}$ is a symmetric chain order, it follows that $\operatorname{Map}_{\alpha}\left(\mathbb{Z}_{n}, \mathcal{P}\right)$ is a symmetric chain order. Also note that $C_{a_{1}} \times \cdots \times C_{a_{n}}$ is a centered subposet of $\operatorname{Map}\left(\mathbb{Z}_{n}, \mathcal{P}\right) / \mathbb{Z}_{n}$. On the other hand, if $\alpha=[\beta, \ldots, \beta]$ is a periodic $n$-bead necklace with labels in $I$, where $\beta=\left(\beta_{1}, \ldots, \beta_{d}\right)$, then:

$$
\left(C_{\beta_{1}} \times \cdots \times C_{\beta_{d}}\right)^{\frac{n}{d}} / \mathbb{Z}_{\frac{n}{d}} \hookrightarrow \operatorname{Map}_{\alpha}\left(\mathbb{Z}_{n}, \mathcal{P}\right) / \mathbb{Z}_{n}
$$

Again, note that this poset is a centered subposet of $\operatorname{Map}\left(\mathbb{Z}_{n}, \mathcal{P}\right) / \mathbb{Z}_{n}$ since it is a cyclic quotient of a centered subposet of $\mathcal{P}^{n}$.

If $d>1$, then $\frac{n}{d}<n$ and $\left(C_{\beta_{1}} \times \cdots \times C_{\beta_{d}}\right)$ is a symmetric chain order, so

$$
\left(C_{\beta_{1}} \times \cdots \times C_{\beta_{d}}\right)^{\frac{n}{d}} / \mathbb{Z}_{\frac{n}{d}}
$$

is a symmetric chain order by induction.

If $d=1$, then:

$$
C^{n} / \mathbb{Z}_{n} \hookrightarrow \operatorname{Map}_{\alpha}\left(\mathbb{Z}_{n}, \mathcal{P}\right) / \mathbb{Z}_{n}
$$

where $C$ is a chain with $m+1$ vertices, for some $m \geq 1$. It suffices to consider the centered subposet $\mathcal{N}(n, m)$. By Lemma 3.2, we have:

$$
\mathcal{N}(n, m) \simeq \mathcal{Q}(m n, m)
$$

If $Q_{\alpha} \subset \mathcal{Q}(m n, m)$, then $\alpha=\left[m a_{1}, \ldots, m a_{s}\right]$, where $a_{1}+\cdots+a_{s}=n$. In particular, note that $s \leq n$. By Lemma 3.3, there are two possibilities for $\mathcal{Q}_{\alpha}$. If $\alpha$ is aperiodic, $\mathcal{Q}_{\alpha}$ is a product of chains, so it is a symmetric chain order. If $\alpha$ is periodic of period $d$, then:

$$
\mathcal{Q}_{[\beta]}^{\frac{s}{d}} / \mathbb{Z}_{\frac{s}{d}} \hookrightarrow Q_{\alpha}
$$


where $[\beta]$ is a $d$-bead aperiodic necklace. In particular, $Q_{[\beta]}$ is itself a product of chains (hence a symmetric chain order). We know that $\beta=\left(m c_{1}, \ldots, m c_{d}\right)$, where $c_{1}+\cdots+c_{d}=$ $\frac{d n}{s}$. There are three possible cases:

(i) If $d>1$, then $\frac{s}{d}<n$. Since $\mathcal{Q}_{[\beta]}$ is a symmetric chain order, by induction we conclude that

$$
\mathcal{Q}_{[\beta]}^{\frac{s}{d}} / \mathbb{Z}_{\frac{s}{d}}
$$

is a symmetric chain order.

(ii) If $d=1$ and $s<n$ then $Q_{[\beta]}$ is a single chain, so $Q_{[\beta]}^{s} / \mathbb{Z}_{s}$ is a symmetric chain order by induction.

(iii) If $d=1$ and $s=n$, then $\beta=(m)$ and $\alpha=[m, \ldots, m]$. In this case:

$$
Q_{[m]}^{n} / \mathbb{Z}_{n} \hookrightarrow Q_{\alpha}
$$

Since $\mathcal{Q}_{[m]}$ is a chain with $m-1$ vertices, we see that we have returned to the case of the $\mathbb{Z}_{n}$-quotient of the $n$-fold power of a single chain. However, note that the we have managed to decrease the length of the chain by two, i.e. from $m+1$ vertices to $m-1$ vertices. Now we can again apply Lemma 3.2 and Lemma 3.3 to the centered subposet $\mathcal{N}(n, m-2)$, etc.

Eventually, after we go through this argument enough times, we will eventually reach the case of:

$$
C^{n} / \mathbb{Z}_{n}
$$

where $C$ is a chain with one or two vertices. If $|C|=1$, there is nothing to show. So we are left with the case where $C$ is a chain with two vertices, i.e. the poset of binary necklaces. It suffices to look at the centered subposet $\mathcal{N}(n, 1)$. By Proposition 3.1,

$$
\mathcal{N}(n, 1) \simeq \mathcal{Q}(n)
$$

Again, we consider the subposets $Q_{\alpha}$. As usual, if $\alpha$ is aperiodic then $Q_{\alpha}$ is covered by a product of symmetric chains. If $\alpha=[\beta, \ldots, \beta]$ is periodic of period $d$ then

$$
\mathcal{Q}_{[\beta]}^{\frac{n}{d}} / \mathbb{Z}_{\frac{n}{d}} \hookrightarrow Q_{\alpha}
$$

where $[\beta]$ is an aperiodic $d$-bead necklace and $\mathcal{Q}_{[\beta]}$ is a product of chains. If $d>1$, then $\frac{n}{d}<n$ so

$$
\mathcal{Q}_{[\beta]}^{\frac{n}{d}} / \mathbb{Z}_{\frac{n}{d}}
$$

is a symmetric chain order by induction. Finally, if $\alpha$ is periodic of period $d=1$ then $\alpha$ is an $n$-bead partition necklace of period 1 whose entries sum to $n$, so $\alpha=[1,1, \ldots, 1]$, but this element was explicitly excluded from the set $\mathcal{Q}(n)$.

Example 4.2. Suppose $\mathcal{P}$ is a disjoint union of 3 symmetric chains $C_{1}, C_{2}$, and $C_{3}$. Let $I=\{1,2,3\}$. Then the poset

$$
\operatorname{Map}\left(\mathbb{Z}_{4}, I\right) / \mathbb{Z}_{4}
$$

has three necklaces of period 1, three necklaces of period 2, and 18 aperiodic necklaces. 


$\begin{array}{rrrrrrr}\text { Period 1: } & {[1,1,1,1]} & {[2,2,2,2]} & {[3,3,3,3]} & & & \\ \text { Period 2: } & {[1,2,1,2]} & {[1,3,1,3]} & {[2,3,2,3]} & & & \\ \text { Aperiodic: } & {[1,1,1,2]} & {[1,1,1,3]} & {[2,2,2,3]} & {[1,2,2,2]} & {[1,3,3,3]} & {[2,3,3,3]} \\ & {[1,1,2,2]} & {[1,1,3,3]} & {[2,2,3,3]} & {[1,2,1,3]} & {[2,1,2,3]} & {[3,1,3,2]} \\ & {[1,1,2,3]} & {[1,1,3,2]} & {[2,2,1,3]} & {[2,2,3,1]} & {[3,3,1,2]} & {[3,3,2,1]}\end{array}$

Therefore $\operatorname{Map}\left(\mathbb{Z}_{4}, \mathcal{P}\right) / \mathbb{Z}_{4}$ can be decomposed as a disjoint union of three types of centered subposets:

$$
\begin{gathered}
\bigsqcup_{i=1}^{3} C_{i}^{4} / \mathbb{Z}_{4} \\
\bigsqcup_{1 \leq i<j \leq 3}\left(C_{i} \times C_{j}\right)^{2} / \mathbb{Z}_{2} \\
\bigsqcup_{\substack{\left.i_{1}, i_{2}, i_{3}, i_{4}\right] \\
\text { aperiodic }}} C_{i_{1}} \times C_{i_{2}} \times C_{i_{3}} \times C_{i_{4}}
\end{gathered}
$$

In the third case we have a product of chains, which has a symmetric chain decomposition by the Greene-Kleitman rule. Similarly, $C_{i} \times C_{j}$ also has a symmetric chain decomposition and the number of chains is equal to $\min \left(\left|C_{i}\right|,\left|C_{j}\right|\right)$. The components in the second case will be of the form $C \times C^{\prime}$ or $C^{2} / \mathbb{Z}_{2}$ for some chains $C$ and $C^{\prime}$. In each case, we are reduced to the case of cyclic quotients of powers of a single chain.

Example 4.3. Let $C=\{0<1<2<3<4\}$ be a chain with 5 vertices. We will construct a symmetric chain decomposition of the poset of 6 -bead 5 -ary necklaces:

$$
\operatorname{Map}\left(\mathbb{Z}_{6}, C\right) / \mathbb{Z}_{6}
$$

By removing the necklaces $[0,0,0,0,0,0]$ and $[4,4,4,4,4,4]$, we get the centered subposet:

$$
\mathcal{N}(6,4) \simeq \mathcal{Q}(24,4)
$$

We can ignore any aperiodic $\alpha$ since the corresponding $\mathcal{Q}_{\alpha}$ will covered by a product of chains. So we need to list all the periodic fundamental partition necklaces $\alpha$ whose entries add up to 24 and are all divisible by 4 . They are:

$$
[24] \quad[12,12] \quad[8,8,8] \quad[4,8,4,8] \quad[4,4,4,4,4,4] \text {. }
$$

Now $Q_{[24]}$ is a chain, and three of the others are covered by a poset of the form $P^{d} / \mathbb{Z}_{d}$ where $P$ is a chain or a product of chains and $d$ is a proper divisor of 6 :

$$
\mathcal{Q}_{[12]}^{2} / \mathbb{Z}_{2} \hookrightarrow \mathcal{Q}_{[12,12]} \quad Q_{[8]}^{3} / \mathbb{Z}_{3} \hookrightarrow \mathcal{Q}_{[8,8,8]} \quad\left(\mathcal{Q}_{[4]} \times \mathcal{Q}_{[8]}\right)^{2} / \mathbb{Z}_{2} \hookrightarrow \mathcal{Q}_{[4,8,4,8]} .
$$

The final poset still involves $\mathbb{Z}_{6}$ but it involves a chain which has $5-2=3$ vertices:

$$
\mathcal{Q}_{[4]}^{6} / \mathbb{Z}_{6} \hookrightarrow \mathcal{Q}_{[4,4,4,4,4,4]}
$$


Note that $Q_{[4]}^{6} / \mathbb{Z}_{6}$ is the poset of 6 -bead ternary necklaces. Removing the extremal elements, we get the poset:

$$
\mathcal{N}(6,2) \simeq \mathcal{Q}(12,2)
$$

whose periodic fundamental partition necklaces are:

$$
[12] \quad[6,6] \quad[4,4,4] \quad[2,4,2,4] \quad[2,2,2,2,2,2] \text {. }
$$

As before, the first four posets can be dealt with inductively, and the final poset:

$$
\mathcal{Q}_{[2,2,2,2,2,2]} \simeq \mathcal{Q}_{[2]}^{6} / \mathbb{Z}_{6}
$$

has exactly one element.

Acknowledgements. I would like to thank the Department of Mathematics at Michigan State University for their hospitality. I am especially grateful to Bruce Sagan for his encouragement while this project was under way. This paper also benefited greatly from several referee comments.

\section{References}

[1] N. G. de Bruijn, Ca. van Ebbenhorst Tengbergen, and D. Kruyswijk. On the set of divisors of a number. Nieuw Arch. Wiskunde (2), 23:191-193, 1951.

[2] Dwight Duffus, Jeremy McKibben-Sanders, and Kyle Thayer. Some Quotients of the Boolean Lattice are Symmetric Chain Orders. http://arxiv.org/abs/1107.1098.

[3] Curtis Greene and Daniel J. Kleitman. Strong versions of Sperner's theorem. J. Combinatorial Theory Ser. A, 20(1):80-88, 1976.

[4] Jerrold R. Griggs, Charles E. Killian, and Carla D. Savage. Venn diagrams and symmetric chain decompositions in the Boolean lattice. Electron. J. Combin. 11 (2004).

[5] Patricia Hersh and Anne Schilling. Symmetric chain decomposition for cyclic quotients of Boolean algebras and relation to cyclic crystals. http://arxiv.org/abs/1107.4073.

[6] Kelly Kross Jordan. The necklace poset is a symmetric chain order. J. Combin. Theory Ser. A 117 (2010), no. 6, 625-641. 